\title{
Towards efficient methods to construct bis-oxa/thiazoles
}

\author{
Lucía Landeira, Florencia Parpal, Eduardo Manta, Gloria Serra, Laura Scarone \\ Facultad de Química, UDELAR, Gral. Flores 2124, Montevideo 11800, Uruguay \\ *e-mail: laurito@fq.edu.uy
}

Keywords: bis-oxazole/thiazole, oxazol-thiazole, bioactive marine products.

\section{INTRODUCTION}

Natural products play an important role in drug development, particularly in anticancer, antibiotics and antiparasitics drugs. ${ }^{1}$ [2,4'] or [2,5'] Bis-1,3oxa/thia-aza scaffolds are present in numerous structures of marine natural products with interesting biological actvities. ${ }^{2}$ As examples, we cited Bengazoles, Laucamides and Largazole.

As part of our search for compounds as candidates for anticancer or antiparasitic drugs employing molecular simplification, ${ }^{3}$ we are interested in an efficient methodology to synthesize bis-1,3oxa/thiaaza like bis-thiazole (1, figure 1) and bisoxazoles or oxazol-thiazole (2, figure 1$)$.

Figure 1: Bis-1,3-oxa/thiaza systems
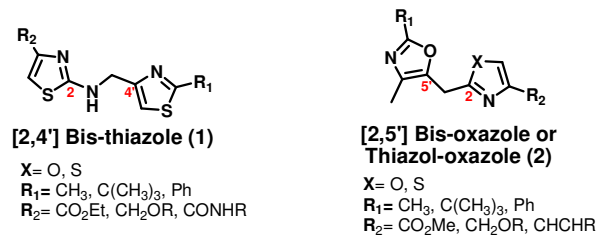

\section{RESULTS AND DISCUSSION}

Bis-thiazoles of type 1, were prepared as is shown in scheme 1 employing Hantzsch's methodology to obtain thiazole 6 and then, using coupling agents to prepare the amide 7 . The reduction method reported by Kuehne, ${ }^{4}$ allowed us to obtain the thiazoles linked by a methylenamine bridge.

Scheme 1: Synthesis of [2,4'] bis-thiazole.

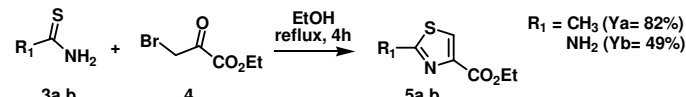

$$
\begin{aligned}
& \text { - } \\
& 5 a \\
& 6 a \quad 5 b
\end{aligned}
$$

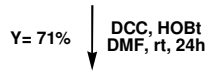

$$
\begin{aligned}
& \text { - }
\end{aligned}
$$

In order to prepare bis-oxazoles or oxazol-thiazole of type 2, we started synthesizing the trisubstituted oxazole 11 employing cyclocondensation cascade of oximes and acyl chlorides as was described by
Wipf. ${ }^{5}$ Using ethyl levulinate 9 as starting material it was possible to obtain only oxazoles containing an ester group with aromatic at $R_{2}$ substituent. Then, ester 11 was hydrolized to the carboxilic acid 12 and coupled with L-serine methyl ester. (Scheme 2)

Scheme 2: Synthesis of [2,5'] bis-oxazoles or [2,5'] thhiazol-oxazole.

$$
\text { (1) }
$$

The amides 13 are key intermediates to obtain [2,5'] bisoxazole 14 by cyclodehydration and oxidation process or [2,5'] thiazole-oxazole 15 by using Lawesson reagent and then cyclodehydative agent and oxidation reactions.

Compounds 7, 8, 14 and 15 will be submitted to anthelmintic assay.

\section{CONCLUSION}

The methodology to prepare bis-thiazoles (1) results a rapid and efficient strategy to generate molecular diversity by using different starting amides or by modifying bis-thiazol 8 on the ester group.

We are searching an alternative synthetic route to prepare a wide variety of oxazole 11 , so that allows us synthesized diversity at [2,5'] bis-1,3-oxa/thiaaza systems.

\section{ACKNOWLEDGEMENTS}

Facultad de Química, CSIC, Pedeciba, ANII. Lab. RMN, MS, LEA.

\section{REFERENCES}

Newman, D. J.; Cragg, G. M. J. Nat. Prod. 2007, 70, 461

2 Jin, Z. Nat. Prod. Rep. 2006, 23, 464.

3 Scarone, L.; Fajardo, J.; Saldaña, J.; Domínguez, L.; Espósito, P.; Dematteis, S.; Wipf, P.; Manta, E.; Serra, G. Lett. Drug, Des. \& Disc. 2009,

6,413 and references cited there.

${ }^{4}$ Kuehne, M.E.; Shannon, P.J. J. Org. Chem. 1977, 42, 2082

${ }^{5}$ Wipf, P.; Fletcher, J.M.; Scarone, L. Tetrahedron Lett 2005, 46, 5463. 